\title{
An analogue computer for pressure-time measurements in valvular heart disease
}

\author{
I R WILLIAMSON AND A H KITCHIN
}

From the Department of Medical Physics and Engineering and Department of Medicine, Western General Hospital, Edinburgh

ABSTRACT Accurate measurement of pressure differences across a diseased heart valve involveș either laborious planimetry or elaborate digital computing facilities. An analogue device is described, simple and inexpensive to construct, which derives from the recorder input of twopressure signals the time (seconds/minute) of valve opening and the mean pressure differencen during this time. Measurements may be repeated over long periods. The importance of using? pressure differences as compared with peak or end diastolic gradients is noted; serious errors in assessment of valvular disease may otherwise occur.

The selection of patients for heart valve surgery depends, in the great majority of cases, on accurate haemodynamic measurements made at cardiac catheterisation. Non-invasive methods of diagnosis, in particular echocardiography, play an increasing role in demonstrating disordered structure and movement of valvular tissue and ventricular wall. Since, however, they do not measure haemodynamic function in quantitative terms they are insufficiently precise and may be misleading. ${ }^{1}$ A simultaneous recording of oscillatory pressures upstream and downstream to the diseased valve remains a crucial part of assessment in valve disease. The methods used subsequently to analyse the pressure difference across the valve vary widely from casual inspection of the aortic peak pressure gradient or mitral end diastolic gradient, to highly automated processing of the signals by digital computer.

We describe a simple analogue computer for attachment to a chart recorder which will give an immediate read-out of mean forward pressure differences over periods of up to 30 seconds and of the time intervals (in $\mathrm{sec} / \mathrm{min}$ ) of valve opening during which the pressure calculation is made. It thus gives an immediate read-out of two of three variables required for calculation of the Gorlin formula. ${ }^{2}$

The computer is shown in fig 1 and a block diagram of the constituent parts in fig 2 .

Address for reprint requests: Dr AH Kitchin, Department of Medicine, Western General Hospital, Crewe Road, Edinburgh EH4 2XU.

\section{Operation}

Two operating ranges are included $-0-40 \mathrm{mmHg}$ and $0-100 \mathrm{mmHg}$. The output signals from the two pressure gauges are accurately balanced a? the zero and standard pressures of the appropriate range. Switches are pressed to start and stop the ${ }^{3}$ computer. It is convenient to set it up in paralle with a chart recorder so that a visual record may. be obtained occasionally to check the operation of the computer.

The differential amplifier (see fig 2) is used tơ derive $\mathbf{P}_{1}-\mathbf{P}_{2}$, the instantaneous differential blooc pressure signal. The input polarity switch is set so that $\mathbf{P}_{1}-\mathbf{P}_{2}$ is positive when the valve is open. Wher? the start switch is pressed, three integrations are started. These are described below.

$\mathbf{P}_{1}-\mathbf{P}_{2}$ is integrated, when positive, by the press $\frac{P}{8}$ ure integrator. The polarity of $\mathbf{P}_{1}-\mathbf{P}_{2}$ is determinec. by the polarity detector which in turn controls this integrator. Thus the output of the pressure integrator increases in steps as the computeR operates.

The forward time integrator (again controlled by the polarity detector) integrates those times when $P_{1}-P_{2}$ is positive. It uses a time reference signal at its input. Its output also increases in steps.

The total time integrator uses the same tim reference signal but its output steadily increases as the computer operates.

To avoid inaccuracy, the start and stop circuitre ensures that an integral number of cardiac cycles 


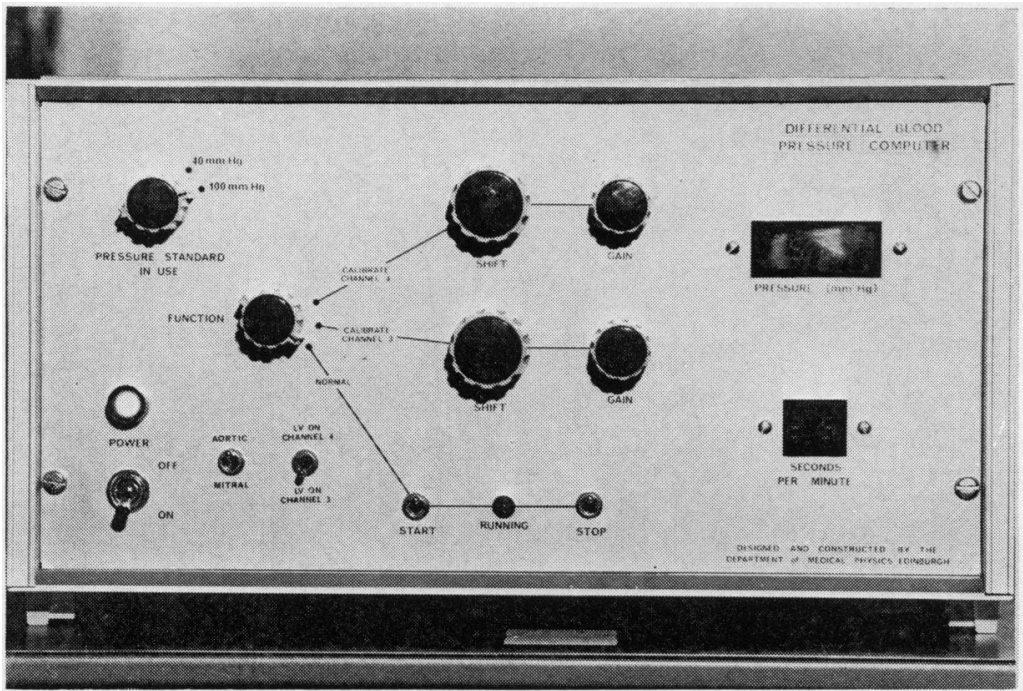

Fig 1 The computer used in conjunction with an ultraviolet chart recorder.

Fig 2 Block diagram of constituent parts.

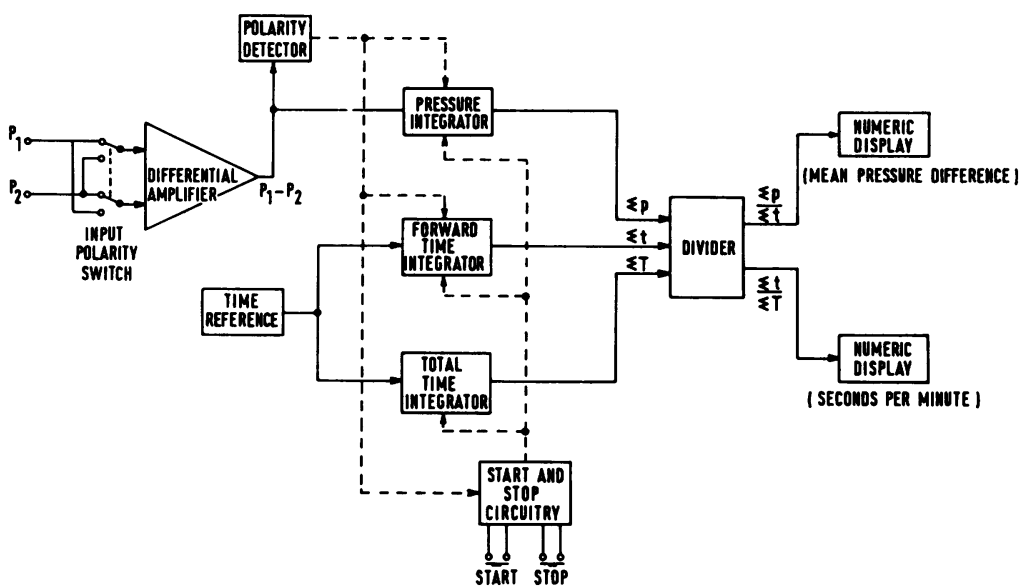

is included in all three integrations. The polarity detector is used to determine the end of each cycle.

When the stop switch is pressed, the final values of the above integrals-called $\leqslant \mathrm{p}, \leqslant \mathrm{t}$, and $\leqslant \mathrm{T}$ respectively-are used by the divider to derive $\leqslant \mathrm{p} / \xi \mathrm{t}$, the mean pressure difference, and $\leqslant \mathrm{t} /$ $\leqslant \mathrm{T}$, the seconds per minute figure. These results are displayed on the instrument and the three integrators are reset to zero ready for the next calculation.

\section{Evaluation}

Simultaneous measurements of valvular pressure gradients by the computer and by direct planimetry of superimposed pressures recorded on 30 $\mathrm{cm}$ wide ultraviolet paper at a paper speed of 135 $\mathrm{mm} / \mathrm{sec}$ (sensitivity $4 \mathrm{~mm} / \mathrm{mmHg}$ pressure) have been carried out in 12 patients. In all cases the pressure gradients agreed to within $3 \mathrm{mmHg}$ in the case of aortic valve gradients, and $1 \mathrm{mmHg}$ in the case of mitral valve gradients. Small pressure differences may be difficult to measure if the signals are noisy. If measurements are made of very small pressure gradients across the mitral valve in diastole the diastolic filling period may be artificially cut short by crossing of the pressure traces, given an unduly low diastolic filling period. This may be corrected either visually or by making a deliberate $5 \mathrm{mmHg}$ offset to separate the traces which must of course be subtracted in the calculation. 
The computer is now in routine use in the laboratory.

\section{Discussion}

The importance of accuracy in the measurement of mean simultaneous pressure differences is shown in fig 3 in which the peak pressure differences in 36 cases of aortic stenosis are plotted against the true mean pressure difference obtained by planimetry of the records (fig 4). It is clear that the peak pressure difference may bear little resemblance to the true one. The same applies in the case of mitral diastolic gradients.

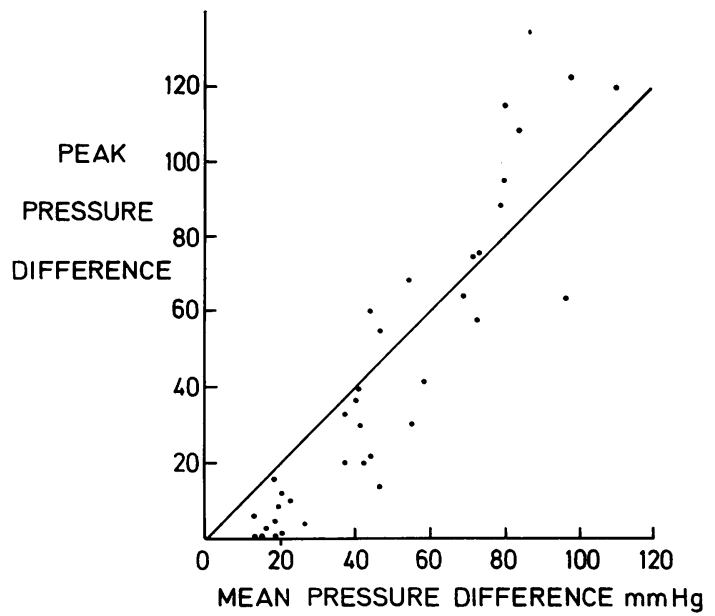

Fig 3 Relation of peak to mean systolic pressure difference in 36 consecutive cases of aortic valve disease.

Calculation of valve area has frequently become discredited through inappropriate application of hydraulic formulae in cases where the variables are measured with poor precision and frequently without correction for regurgitant flow. Yet it is clear that there is no other method of measuring, in aortic stenosis for example, the true obstructive load imposed by the valve. Figure 5 shows the poor correlation between pressure difference across the aortic valve and calculated valve area. It is clear that without the valve area calculation many patients would be operated on unnecessarily, and some with small pressure gradients and low flow would be denied operation despite the severity of the aortic obstruction. Apparent poor ventricular function may be caused either by poor contractility or by the effects of a greatly increased afterload, relief of which would restore normal function. The need for precision in haemodynamic measurement is obvious.

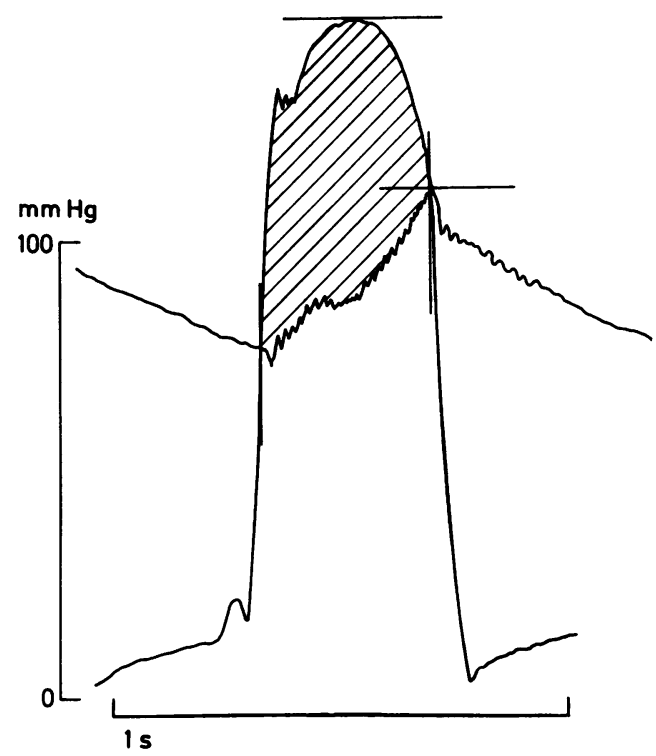

Fig 4 Left ventricular and aortic pressure pulses in aortic stenosis; relation of peak and instantaneous systolic pressure differences. Shaded area is pressure difference across the valve during left ventricular ejection.

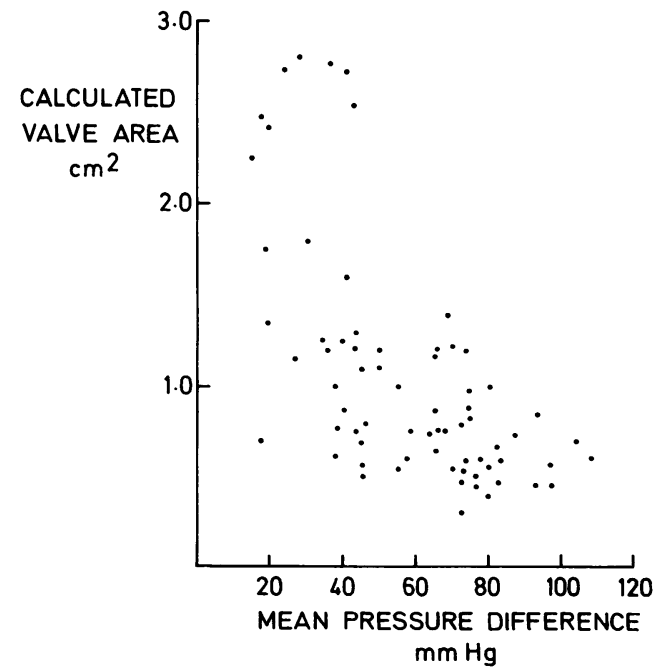

Fig 5 Relation of calculated valve area and mean systolic pressure difference in 69 cases of aortic stenosis. In the valve area calculation regurgitation flow was estimated by indicator dilution methods. 
The advantages claimed for this instrument are that it eliminates the need for much time-consuming analysis of records by planimetry, and permits measurement of valve gradient and time intervals over longer periods than normal or repeated measurements at intervals to assess, for example, the effect of drugs or exercise. The results are immediately available and can be checked by comparison with a simultaneous recording. Finally, it is robust and relatively inexpensive to construct from parts costing approximately $£ 300$. It is not available commercially but a circuit diagram may be obtained from the authors.

\section{References}

1 Cope GD, Kisslo JA, Johnson ML, Behar VS. A reassessment of the echocardiogram in mitral stenosis. Circulation 1975; 52:664-70.

2 Gorlin R, Gorlin SG. Hydraulic formula for calculation of area of stenotic mitral valve, other cardiac valves and central circulatory shunts. Am Heart J 1951; 41:1-29. 